\title{
EXTREME SPECTRA REALIZATION BY REAL SYMMETRIC TRIDIAGONAL AND REAL SYMMETRIC ARROW MATRICES*
}

\author{
HUBERT PICKMANN $^{\dagger}$, JUAN C. EGAÑA ${ }^{\ddagger}$, AND RICARDO L. SOTO ${ }^{\S}$
}

Abstract. We consider the following two problems: to construct a real symmetric arrow matrix $A$ and to construct a real symmetric tridiagonal matrix $A$, from a special kind of spectral information: one eigenvalue $\lambda^{(j)}$ of the $j \times j$ leading principal submatrix $A_{j}$ of $A, j=1,2, \ldots, n$; and one eigenpair $\left(\lambda^{(n)}, \mathbf{x}\right)$ of $A$. Here we give a solution to the first problem, introduced in [J. Peng, X.Y. Hu, and L. Zhang. Two inverse eigenvalue problems for a special kind of matrices. Linear Algebra Appl., 416:336-347, 2006.]. In particular, for both problems to have a solution, we give a necessary and sufficient condition in the first case, and a sufficient condition in the second one. In both cases, we also give sufficient conditions in order that the constructed matrices be nonnegative. Our results are constructive and they generate algorithmic procedures to construct such matrices.

Key words. Real symmetric tridiagonal matrices, Real symmetric arrow matrices, Eigenproblem.

AMS subject classifications. $65 \mathrm{~F} 15,65 \mathrm{~F} 18,15 \mathrm{~A} 18$.

1. Introduction. A discrete undamped system, consisting of $n$ masses $m_{i}>$ 0 connected by $n$ springs of stiffness $k_{i}>0$, is characterized by the generalized eigenvalue problem:

$$
\left(K_{n}-\lambda M_{n}\right) \mathbf{u}=0
$$

where $\lambda=\omega^{2}$, with $\omega$ a natural frequency and $u$ the respective vibration mode, while $M_{n}=\operatorname{diag}\left\{m_{1}, m_{2}, \ldots, m_{n}\right\}$ and

$$
K_{n}=\left[\begin{array}{ccccc}
k_{1}-k_{2} & -k_{2} & & & \\
-k_{2} & k_{2}+k_{3} & -k_{3} & & \\
& \ddots & \ddots & \ddots & \\
& & -k_{n-1} & k_{n-1}+k_{n} & -k_{n} \\
& & & -k_{n} & k_{n}
\end{array}\right]
$$

\footnotetext{
* Received by the editors on January 20, 2011. Accepted for publication on July 10, 2011. Handling Editors: Michael Neumann and Xingzhi Zhan.

†Departamento de Matemáticas, Universidad de Tarapacá, Arica, Chile (hpickmanns@ucn.cl). Supported by Proyect UTA 4730-11, Chile.

¥Departamento de Matemáticas, Universidad Católica del Norte, Antofagasta, Casilla 1280, Chile (jegana@ucn.cl). Supported by Project DGIP-UCN, Chile.

$\S$ Departamento de Matemáticas, Universidad Católica del Norte, Antofagasta, Casilla 1280, Chile (rsoto@ucn.cl). Supported by Fondecyt 1085125, Chile.
} 
are the mass matrix and the stiffness matrix, respectively.

The generalized eigenvalue problem (1.1) can be expressed as the standard eigenvalue problem

$$
\left(J_{n}-\lambda I_{n}\right) \mathbf{x}=0
$$

where $J_{n}=M_{n}^{-\frac{1}{2}} K_{n} M_{n}^{-\frac{1}{2}}$ and $x=M_{n}^{\frac{1}{2}} \mathbf{u}$. It is well known that the system $\left(M_{n}, K_{n}\right)$ can be reconstructed from some spectral information of the matrix $J_{n}$. There are several procedures to reconstruct $J_{n}$. One of the most popular procedures considers the $n$ eigenvalues of $J_{n}$, and the $n-1$ eigenvalues of the submatrix $J_{n-1}$, obtained by deleting the last (first) row and column of $J$ (see [5]).

Recently, new spectral information to reconstruct the system $\left(M_{n}, K_{n}\right)$ have been considered by Huang et al. in [7]:

i. $\lambda_{i}$ and $\lambda_{i}^{*}$, associate to the maximal and minimal natural frequencies of $\left(M_{i}, K_{i}\right), i=1, \ldots, n$, respectively.

ii. $\lambda_{i} \in \mathbb{R}^{+}$, associate to the maximal (or minimal) frequency of $\left(M_{i}, K_{i}\right), i=$ $1, \ldots, n$, and $x \in \mathbb{R}^{n}$, the mode corresponding to $\lambda_{n}$,

where $\left(M_{i}, K_{i}\right)$ is the subsystem of $\left(M_{n}, K_{n}\right)$, that is, obtained by fixing the mass $m_{i+1}$. Note that the subsystems $\left(M_{i}, K_{i}\right)$ correspond to the leading principal submatrices of $J_{n}$.

Peng et al. [8] propose two inverse eigenvalue problems to reconstruct an arrow matrix from similar spectral data. Unfortunately, the proposed solutions in [8] are not correct. In [9], a correct solution is given for this problem, while in [10], a Jacobi matrix is reconstructed from the same spectral data.

In this paper, we study two inverse eigenproblems. One of them related with the construction of an $n \times n$ symmetric arrow matrix of the form

$$
\left[\begin{array}{ccccc}
a_{1} & b_{1} & b_{2} & \cdots & b_{n-1} \\
b_{1} & a_{2} & & & \\
b_{2} & & a_{3} & & \\
\vdots & & & \ddots & \\
b_{n-1} & & & & a_{n}
\end{array}\right], \quad a_{j}, b_{j} \in \mathbb{R}
$$

and the other one related with the construction of an $n \times n$ symmetric tridiagonal 
matrix of the form

$$
\left[\begin{array}{ccccc}
a_{1} & b_{1} & & & \\
b_{1} & a_{2} & b_{2} & & \\
& b_{2} & a_{3} & \ddots & \\
& & \ddots & \ddots & b_{n-1} \\
& & & b_{n-1} & a_{n}
\end{array}\right], \quad a_{j}, b_{j} \in \mathbb{R} .
$$

The following eigenproblem was proposed and discussed in [8, Problem II]:

Problem 1.1. Given $n$ real numbers $\lambda^{(1)}, \lambda^{(2)}, \ldots, \lambda^{(n)}$, and a real vector $\mathbf{x}=$ $\left(x_{1}, x_{2}, \ldots, x_{n}\right)^{T}$, find an $n \times n$ symmetric arrow matrix $A$ of the form (1.2), where the $a_{i}$ are all distinct for $i=2,3, \ldots, n$ and the $b_{i}$ are all positive, and such that $\lambda^{(j)}$ is an eigenvalue of the $j \times j$ leading principal submatrix $A_{j}$ of $A, j=1,2, \ldots, n$, and $\left(\lambda^{(n)}, \mathbf{x}\right)$ is an eigenpair of $A$.

We observe that in this problem, the formulae to compute the $a_{i}$ and $b_{i}$, given in [8, Theorem 2], may lead us to a matrix which does not satisfy the requirements. For instance, the numbers

$$
\begin{array}{cccc}
\lambda^{(1)} & \lambda^{(2)} & \lambda^{(3)} & \lambda^{(4)} \\
2 & \frac{(5-\sqrt{65})}{2} & -2 & \frac{(5+\sqrt{85})}{2}
\end{array}
$$

and the vector

$$
\mathbf{x}=\left(\frac{1}{2}(\sqrt{85}-1), 4,2,1\right)^{T}
$$

satisfy the conditions in [8, Theorem 2]. However, the unique resultant matrix is

$$
A=\left[\begin{array}{llll}
2 & 4 & 2 & 1 \\
4 & 3 & 0 & 0 \\
2 & 0 & 3 & 0 \\
1 & 0 & 0 & 3
\end{array}\right]
$$

which has repeated diagonal entries.

In this work, we rediscuss the above eigenproblem to give a necessary and sufficient condition, which leads us to a correct solution. We also study the following eigenproblem for real symmetric tridiagonal matrices:

Problem 1.2. Given $n$ real numbers $\lambda^{(j)}, j=1,2, \ldots, n$ and a real vector $\mathbf{x}=\left(x_{1}, \ldots, x_{n}\right)^{T}$, find necessary and sufficient conditions for the existence of an $n \times n$ symmetric tridiagonal matrix $A$ of the form (1.3), such that $\lambda^{(j)}$ is an eigenvalue of the $j \times j$ leading principal submatrix $A_{j}$ of $A, j=1,2, \ldots, n$, and $\left(\lambda^{(n)}, \mathbf{x}\right)$ is an eigenpair of $A$. 
This family of matrices appears in certain symmetric inverse eigenvalue and inverse Sturm-Liouville problems, which arise in many applications, including modern control theory and vibration analysis [1]-[10].

We shall denote as $I_{j}$ the identity matrix of order $j$; as $A_{j}$ the $j \times j$ leading principal submatrix of $A$; as $P_{j}(\lambda)$ the characteristic polynomial of $A_{j}$, and as $\lambda^{(j)}$ an eigenvalue of $A_{j} . \sigma\left(A_{j}\right)$ will denote the spectrum of $A_{j}$.

The paper is organized as follows: In Section 2, we consider Problem 1.1 with $\lambda^{(j)}$ as the maximal eigenvalue of $A_{j}$, and in this case, we give a correct solution to the eigenproblem in $[8$, Problem II]. We also give sufficient conditions in order that the symmetric arrow solution matrix is nonnegative. In Section 3, we consider Problem 1.2 with $\lambda^{(j)}$ being the maximal eigenvalue of $A_{j}$, and give sufficient conditions for the problem to have a solution with all its $b_{i}$ entries positive and also a nonnegative solution. Finally, in Section 4, we show some examples to illustrate the results. Our results are constructive, in the sense that they generate an algorithmic procedure to construct the solution matrices.

2. Solution to Problem 1.1. We recall the following well known lemmas:

Lemma 2.1. Let $A$ be an $n \times n$ matrix of the form (1.2) and let $P_{j}(\lambda)$ the characteristic polynomial of the $j \times j$ leading principal submatrix $A_{j}, j=1,2, \ldots, n$, of $A$. Then the sequence $\left\{P_{j}(\lambda)\right\}_{j=1}^{n}$ satisfies the recurrence relation:

$$
\begin{aligned}
& P_{1}(\lambda)=\left(\lambda-a_{1}\right), \\
& P_{2}(\lambda)=\left(\lambda-a_{2}\right) P_{1}(\lambda)-b_{1}^{2}, \\
& P_{j}(\lambda)=\left(\lambda-a_{j}\right) P_{j-1}(\lambda)-b_{j-1}^{2} \prod_{i=2}^{j-1}\left(\lambda-a_{i}\right), \quad j=3,4, \ldots, n .
\end{aligned}
$$

This recurrence relation can be written as:

$$
P_{j}(\lambda)=\prod_{i=1}^{j}\left(\lambda-a_{i}\right)-\sum_{k=1}^{j-1}\left(b_{k}^{2} \prod_{\substack{i=2 \\ i \neq k+1}}^{j}\left(\lambda-a_{i}\right)\right), \quad j=1,2, \ldots, n .
$$

LEMMA 2.2. [8] Let $A$ be an $n \times n$ matrix of the form (1.2) with all its diagonal entries $a_{j}$ distinct, $j=2,3, \ldots, n$, and all its entries $b_{i}$ positive, $i=1,2, \ldots, n-1$. Then

i) the eigenvalues $\lambda_{1}, \lambda_{2}, \ldots, \lambda_{n}$ of $A$ are all simple,

ii) the zeros of $P_{j-1}(\lambda)$ strictly separate those of $P_{j}(\lambda)$.

Lemma 2.3. [9] Let $P(\lambda)$ be a monic polynomial of degree $n$ with all real zeroes. If $\lambda_{1}$ and $\lambda_{n}$ are, respectively, the minimal and maximal zero of $P(\lambda)$, then 
1. If $\mu<\lambda_{1}$, we have $(-1)^{n} P(\mu)>0$,

2. If $\mu>\lambda_{n}$, we have $P(\mu)>0$.

LEMmA 2.4. [9] Let $A$ be a matrix of the form (1.2) with $b_{i} \neq 0, i=1,2, \ldots, n-1$. Let $\lambda_{1}^{(j)}$ and $\lambda_{j}^{(j)}$, respectively, be the minimal and the maximal eigenvalue of the leading principal submatrix $A_{j}, j=1,2, \ldots, n$, of $A$. Then

$$
\lambda_{1}^{(j)}<\cdots<\lambda_{1}^{(3)}<\lambda_{1}^{(2)}<\lambda_{1}^{(1)}<\lambda_{2}^{(2)}<\lambda_{3}^{(3)}<\cdots<\lambda_{j}^{(j)} .
$$

LEMMA 2.5. [8] Let $\mathbf{x}_{1}, \mathbf{x}_{2}, \ldots, \mathbf{x}_{n}$ be a set of orthonormal eigenvectors associated to the eigenvalues $\lambda_{1}, \lambda_{2}, \ldots, \lambda_{n}$ of an $n \times n$ matrix $A$ of the form (1.2), with all its diagonal entries $a_{j}$ distinct, $j=2,3, \ldots, n$, and all its entries $b_{i}$ positive, $i=$ $1,2, \ldots, n-1$. Then $x_{\mu j} \neq 0$ for $\mu, j=1,2, \ldots, n$, where $x_{\mu j}$ denotes the $\mu$-th entry of the vector $\mathbf{x}_{j}$.

Now we propose a solution to Problem 1.1.

THEOREM 2.6. Given $n$ real numbers $\lambda^{(1)}<\lambda^{(2)}<\cdots<\lambda^{(n)}$ and a real vector $\mathbf{x}=\left(x_{1}, \ldots, x_{n}\right)^{T}$, then there exists a matrix $A$ of the form (1.2), with all its diagonal entries $a_{j}$ distinct, $j=2,3, \ldots, n$, and all its entries $b_{i}$ positive, $i=1,2, \ldots, n-1$, in such a way that $\lambda^{(j)}$ is the maximal eigenvalue of the $j \times j$ leading principal submatrix $A_{j}, j=1,2, \ldots, n$, and $\left(\lambda^{(n)}, \mathbf{x}\right)$ is an eigenpair of $A$ if and only if the following conditions are satisfied:

$$
\begin{gathered}
x_{1} x_{i}>0, \quad i=1, \ldots, n, \\
\frac{x_{1}^{2}}{x_{2}^{2}} \geq \frac{4\left(\lambda^{(n)}-\lambda^{(2)}\right)}{\lambda^{(2)}-\lambda^{(1)}}, \\
\frac{x_{1}^{2}}{x_{j}^{2}} \geq \frac{4\left(\lambda^{(n)}-\lambda^{(j)}\right) \prod_{i=2}^{j-1}\left(\lambda^{(j)}-a_{i}\right)}{P_{j-1}\left(\lambda^{(j)}\right)}, \quad j=3,4, \ldots, n,
\end{gathered}
$$

and

$$
\frac{x_{k+1}}{x_{j+1}} \neq \frac{b_{k}}{b_{j}}, \quad j=2,3, \ldots, n-1 \text { and } k=1,2, \ldots, j-1
$$

Proof. First, we observe that to prove the existence of a matrix $A$ with the required spectral properties is equivalent to show that the system of equations

$$
\begin{aligned}
P_{j}\left(\lambda^{(j)}\right) & =0, \quad j=1,2, \ldots, n, \\
A \mathbf{x} & =\lambda^{(n)} \mathbf{x},
\end{aligned}
$$


has real solutions $a_{j}$ and $b_{j-1}$, such that $a_{j} \neq a_{k}, j \neq k$ and $b_{j-1}>0$.

From (2.6), it is clear that

$$
a_{1}=\lambda^{(1)}
$$

For $j=2,3, \ldots, n$, the entries $a_{j}$ and $b_{j-1}$ can be obtained as follows: We rewrite (2.7) as:

$$
\left.\begin{array}{c}
a_{1} x_{1}+b_{1} x_{2}+\cdots+b_{n-1} x_{n}=\lambda^{(n)} x_{1} \\
b_{j-1} x_{1}+a_{j} x_{j}=\lambda^{(n)} x_{j}, j=2,3, \ldots, n
\end{array}\right\} .
$$

From $(2.2)$ we have $x_{i} \neq 0$ for $i=1, \ldots, n$, and then

$$
a_{j}=\lambda^{(n)}-b_{j-1} \frac{x_{1}}{x_{j}}, \quad j=2,3, \ldots, n .
$$

By substituting (2.9) in (2.6), for $j=2$, we obtain the following quadratic equation in $b_{1}$ :

$$
b_{1}^{2}-b_{1} \frac{x_{1}}{x_{2}} P_{1}\left(\lambda^{(2)}\right)+\left(\lambda^{(n)}-\lambda^{(2)}\right) P_{1}\left(\lambda^{(2)}\right)=0
$$

This equation has real solutions

$$
b_{1}=\frac{1}{2}\left[\frac{x_{1}}{x_{2}} P_{1}\left(\lambda^{(2)}\right) \pm \sqrt{\triangle_{1}}\right]
$$

if

$$
\triangle_{1}=\frac{x_{1}^{2}}{x_{2}^{2}}\left[P_{1}\left(\lambda^{(2)}\right)\right]^{2}-4\left(\lambda^{(n)}-\lambda^{(2)}\right) P_{1}\left(\lambda^{(2)}\right) \geq 0,
$$

or, from Lemma 2.2 , if

$$
\frac{x_{1}^{2}}{x_{2}^{2}} \geq \frac{4\left(\lambda^{(n)}-\lambda^{(2)}\right)}{P_{1}\left(\lambda^{(2)}\right)},
$$

which is condition (2.3). Moreover, from Lemma $2.2($ ii) and Lemma 2.4,

$$
4\left(\lambda^{(n)}-\lambda^{(2)}\right) P_{1}\left(\lambda^{(2)}\right)>0
$$

and

$$
\frac{x_{1}}{x_{2}} P_{1}\left(\lambda^{(2)}\right)>\left|\sqrt{\triangle_{1}}\right|
$$

Then, it follows that $b_{1}>0$. 
From Lemma 2.1 , for $j=3,4, \ldots, n$, it follows that

$$
P_{j}\left(\lambda^{(j)}\right)=\left(\lambda^{(j)}-a_{j}\right) P_{j-1}\left(\lambda^{(j)}\right)-b_{j-1}^{2} \prod_{i=2}^{j-1}\left(\lambda^{(j)}-a_{i}\right)=0 .
$$

By substituting (2.9) in (2.10) we obtain the quadratic equation

$$
b_{j-1}^{2} \prod_{i=2}^{j-1}\left(\lambda^{(j)}-a_{i}\right)-b_{j-1} \frac{x_{1}}{x_{j}} P_{j-1}\left(\lambda^{(j)}\right)+\left(\lambda^{(n)}-\lambda^{(j)}\right) P_{j-1}\left(\lambda^{(j)}\right)=0
$$

whose real solutions are given by

$$
b_{j-1}=\frac{\frac{x_{1}}{x_{j}} P_{j-1}\left(\lambda^{(j)}\right) \pm \sqrt{\triangle_{j-1}}}{2 \prod_{i=2}^{j-1}\left(\lambda^{(j)}-a_{i}\right)}
$$

if

$$
\triangle_{j-1}=\frac{x_{1}^{2}}{x_{j}^{2}}\left[P_{j-1}\left(\lambda^{(j)}\right)\right]^{2}-4\left(\lambda^{(n)}-\lambda^{(j)}\right) P_{j-1}\left(\lambda^{(j)}\right) \prod_{i=2}^{j-1}\left(\lambda^{(j)}-a_{i}\right) \geq 0
$$

or, from Lemma 2.2 , if

$$
\frac{x_{1}^{2}}{x_{j}^{2}} \geq \frac{4\left(\lambda^{(n)}-\lambda^{(j)}\right) \prod_{i=2}^{j-1}\left(\lambda^{(j)}-a_{i}\right)}{P_{j-1}\left(\lambda^{(j)}\right)},
$$

which is condition (2.4). Moreover, from Lemma 2.2 and Lemma 4 in [9],

$$
4\left(\lambda^{(n)}-\lambda^{(j)}\right) P_{j-1}\left(\lambda^{(j)}\right) \prod_{i=2}^{j-1}\left(\lambda^{(j)}-a_{i}\right)>0
$$

and

$$
\frac{x_{1}}{x_{j}} P_{j-1}\left(\lambda^{(j)}\right)>\left|\sqrt{\triangle_{j-1}}\right|
$$

Then it follows that $b_{j-1}>0$.

Moreover, from (2.11), (2.1) and (2.9), we have

$$
a_{1} x_{1}+b_{1} x_{2}+\cdots+b_{n-1} x_{n}=a_{1} x_{1}+\sum_{k=1}^{n-2} b_{k} x_{k+1}+b_{n-1} x_{n}
$$




$$
\begin{aligned}
& =a_{1} x_{1}+\sum_{k=1}^{n-2} b_{k} x_{k+1}+\frac{x_{1} P_{n-1}\left(\lambda^{(n)}\right)}{\prod_{i=2}^{n-1}\left(\lambda^{(n)}-a_{i}\right)} \\
& =a_{1} x_{1}+\sum_{k=1}^{n-2} b_{k} x_{k+1}+x_{1} \frac{\prod_{i=1}^{n-1}\left(\lambda^{(n)}-a_{i}\right)-\sum_{k=1}^{n-2}\left(b_{k}^{2} \prod_{\substack{i=2 \\
i \neq k+1}}^{n-1}\left(\lambda^{(n)}-a_{i}\right)\right)}{\prod_{i=2}^{n-1}\left(\lambda^{(n)}-a_{i}\right)} \\
& =a_{1} x_{1}+\sum_{k=1}^{n-2} b_{k} x_{k+1}+x_{1}\left[\left(\lambda^{(n)}-a_{1}\right)-\frac{1}{x_{1}} \sum_{k=1}^{n-2} b_{k} x_{k+1}\right] \\
& =\lambda^{(n)} x_{1} .
\end{aligned}
$$

Thus, the first equation in (2.8) is satisfied.

Finally, from (2.5), we have for $j=2$ and $k=1$,

$$
\frac{x_{2}}{x_{3}} \neq \frac{b_{1}}{b_{2}} .
$$

Then,

$$
a_{3}=\lambda^{(n)}-b_{2} \frac{x_{1}}{x_{3}} \neq \lambda^{(n)}-b_{1} \frac{x_{1}}{x_{2}}=a_{2}
$$

For $j=3,4, \ldots, n-1$,

$$
\frac{x_{k+1}}{x_{j+1}} \neq \frac{b_{k}}{b_{j}}, \quad k=1,2, \ldots, j-1 .
$$

Then,

$$
a_{j+1}=\lambda^{(n)}-b_{j} \frac{x_{1}}{x_{j+1}} \neq \lambda^{(n)}-b_{k} \frac{x_{1}}{x_{k+1}}=a_{k+1}, \quad k=1,2, \ldots, j-1
$$

and the entry $a_{j+1}, j=2,3, \ldots, n-1$ is distinct from each one of entries $a_{2}, a_{3}, \ldots, a_{j}$. Hence, all diagonal entries $a_{i}, i=2,3, \ldots, n$ are distinct. Thus, there exists $A=A_{n}$, of the form (1.2), such that $\lambda^{(j)}, j=1,2, \ldots, n$, is the maximal eigenvalue of the $j \times j$ leading principal submatrix $A_{j}$ of $A$, with $a_{i}$ distinct, $i=2,3, \ldots, n$, and $b_{i}$ positive, $i=1,2, \ldots, n-1$. Besides, as $(2.7)$ holds, $\left(\lambda^{(n)}, \mathbf{x}\right)$ is an eigenpair of $A$.

To show the necessity, observe that condition (2.2) is obtained from Lemma 2.5. The conditions (2.3) and (2.4) comes from the fact that $b_{j}>0$ for $j=1,2, \ldots, n-1$. Finally, since $a_{i}, i=2,3, \ldots, n$, are all distinct and (2.9) holds, condition (2.5) is satisfied. $\square$ 
REMARK 2.7. Theorem 2.6 also holds if we take $\lambda^{(j)}$ as the minimal eigenvalue of $A_{j}$. In this case we must have $\lambda^{(n)}<\cdots<\lambda^{(2)}<\lambda^{(1)}$ and condition (2.2) becomes $x_{1} x_{i}<0, i=1, \ldots, n$. The proof is completely similar.

Now we give sufficient conditions in order that the solution matrix $A$ in Problem 1.1 be nonnegative.

COROllary 2.8. Given $n$ real numbers $\lambda^{(1)}<\lambda^{(2)}<\cdots<\lambda^{(n)}$ and a real vector $\mathbf{x}=\left(x_{1}, \ldots, x_{n}\right)^{T}$, there exists an $n \times n$ nonnegative matrix $A$ of the form (1.2), such that $\lambda^{(j)}$ is the maximal eigenvalue of the $j \times j$ leading principal submatrix $A_{j}, j=1,2, \ldots, n$ and $\left(\lambda^{(n)}, \mathbf{x}\right)$ is an eigenpair of $A$, if the following conditions are satisfied:

$$
\begin{gathered}
x_{1} x_{i}>0, \quad i=1, \ldots, n, \\
\frac{x_{1}^{2}}{x_{2}^{2}} \geq \frac{4\left(\lambda^{(n)}-\lambda^{(2)}\right)}{\lambda^{(2)}-\lambda^{(1)}}, \\
\frac{x_{1}^{2}}{x_{j}^{2}} \geq \frac{4\left(\lambda^{(n)}-\lambda^{(j)}\right) \prod_{i=2}^{j-1}\left(\lambda^{(j)}-a_{i}\right)}{P_{j-1}\left(\lambda^{(j)}\right)}, \quad j=3, \ldots, n, \\
\lambda^{(1)} \geq 0 ; \quad \lambda^{(n)} \geq b_{j-1} \frac{x_{1}}{x_{j}}, \quad j=2,3, \ldots, n .
\end{gathered}
$$

Proof. From the proof of Theorem 2.6, conditions (2.12), (2.13) and (2.14) guarantee that the system of equations (2.6)-(2.7) has real solutions $a_{j}$ and positive solutions $b_{j-1}$. It remains to show the nonnegativity of the diagonal entries $a_{j}$. Clearly, from (2.14) and (2.9), $a_{1}=\lambda^{(1)} \geq 0$ and

$$
a_{j}=\lambda^{(n)}-b_{j-1} \frac{x_{1}}{x_{j}} \geq 0, \quad j=2,3, \ldots, n
$$

3. Solution to Problem 1.2. We start by recalling the following well known result:

Lemma 3.1. Let $A$ be an $n \times n$ symmetric tridiagonal matrix of the form (1.3). Let $A_{j}$ be the $j \times j$ leading principal submatrix of $A$, with characteristic polynomial $P_{j}(\lambda), j=1,2, \ldots, n$. Then the sequence $\left\{P_{j}(\lambda)\right\}_{j=1}^{n}$ satisfies

$$
P_{j}(\lambda)=\left(\lambda-a_{j}\right) P_{j-1}(\lambda)-b_{j-1}^{2} P_{j-2}(\lambda), \quad j=1,2, \ldots, n,
$$


where $P_{0}(\lambda)=P_{-1}(\lambda)=1$ and $b_{0}=0$.

The next result gives sufficient conditions for a solution to Problem 1.2 with $b_{i}>0, i=1,2, \ldots, n-1$.

THEOREM 3.2. Given $n$ real numbers $\lambda^{(1)}<\lambda^{(2)}<\cdots<\lambda^{(n)}$, and a real vector $\mathbf{x}=\left(x_{1}, \ldots, x_{n}\right)^{T}$, there exists an $n \times n$ matrix $A$ of the form (1.3), with positive entries $b_{i}$, such that $\lambda^{(j)}$ is the maximal eigenvalue of the $j \times j$ leading principal submatrix $A_{j}$ of $A, j=1,2, \ldots, n$, and $\left(\lambda^{(n)}, \mathbf{x}\right)$ is an eigenpair of $A$, if the following conditions are satisfied:

$$
x_{i} x_{i+1}>0, \quad i=1,2, \ldots, n
$$

and

$$
\lambda^{(n)}>a_{j}+b_{j-1} \frac{x_{j-1}}{x_{j}}, \quad j=1,2, \ldots, n-1 .
$$

Proof. To show the existence of a matrix $A$ of the form (1.3), with the desired spectral properties, it is equivalent to show that the system of equations

$$
\begin{aligned}
P_{j}\left(\lambda^{(j)}\right) & =0, \quad j=1,2, \ldots, n, \\
A \mathbf{x} & =\lambda^{(n)} \mathbf{x},
\end{aligned}
$$

has real solutions $a_{j}$ and $b_{j-1}>0$, where (3.4) can be written as

$$
\left.\begin{array}{rl}
a_{1} x_{1}+b_{1} x_{2} & =\lambda^{(n)} x_{1} \\
b_{1} x_{1}+a_{2} x_{2}+b_{2} x_{3} & =\lambda^{(n)} x_{2} \\
b_{2} x_{2}+a_{3} x_{3}+b_{3} x_{4} & =\lambda^{(n)} x_{3} \\
\vdots & \vdots \\
b_{j-1} x_{j-1}+a_{j} x_{j}+b_{j} x_{j+1} & =\lambda^{(n)} x_{j} \\
\vdots & \vdots \\
b_{n-2} x_{n-2}+a_{n-1} x_{n-1}+b_{n-1} x_{n} & =\lambda^{(n)} x_{n-1} \\
b_{n-1} x_{n-1}+a_{n} x_{n} & =\lambda^{(n)} x_{n}
\end{array}\right\} .
$$

From (3.1), (3.3) and (3.5), it is clear that $a_{1}=\lambda^{(1)}$,

$$
b_{1}=\left(\lambda^{(n)}-a_{1}\right) \frac{x_{1}}{x_{2}}>0
$$

and

$$
b_{j}=\left(\lambda^{(n)}-a_{j}\right) \frac{x_{j}}{x_{j+1}}-b_{j-1} \frac{x_{j-1}}{x_{j+1}}
$$


$j=2,3, \ldots, n-1$. From $(3.2)$,

$$
\left(\lambda^{(n)}-a_{j}\right) \frac{x_{j+1}}{x_{j+1}}>b_{j-1} \frac{x_{j-1}}{x_{j}}
$$

and

$$
\left(\lambda^{(n)}-a_{j}\right) \frac{x_{j}}{x_{j+1}}>b_{j-1} \frac{x_{j-1}}{x_{j+1}} .
$$

Thus, $b_{j}>0, j=2,3, \ldots, n-1$. Besides, from (3.3) it follows that

$$
P_{j}\left(\lambda^{(j)}\right)=\left(\lambda^{(j)}-a_{j}\right) P_{j-1}\left(\lambda^{(j)}\right)-b_{j-1}^{2} P_{j-2}\left(\lambda^{(j)}\right)=0,
$$

$j=2,3, \ldots, n$, and

$$
a_{j}=\lambda^{(j)}-b_{j-1}^{2} \frac{P_{j-2}\left(\lambda^{(j)}\right)}{P_{j-1}\left(\lambda^{(j)}\right)}, j=2,3, \ldots, n .
$$

Observe that from Lemma 2.3 we have $P_{j-1}\left(\lambda^{(j)}\right)>0$.

To show that the last equation in (3.5) is satisfied, we first show that

$$
b_{j}=\frac{x_{j}}{x_{j+1}} \frac{P_{j}\left(\lambda^{(n)}\right)}{P_{j-1}\left(\lambda^{(n)}\right)}, \quad j=1,2, \ldots, n-1,
$$

where $P_{j-1}\left(\lambda^{(n)}\right)>0$ because of Lemma 2.3. For $j=1$, we have

$$
b_{1}=\frac{x_{1}}{x_{2}}\left(\lambda^{(n)}-a_{1}\right)=\frac{x_{1}}{x_{2}} \frac{P_{1}\left(\lambda^{(n)}\right)}{P_{0}\left(\lambda^{(n)}\right)} .
$$

Now, suppose that (3.7) is true for $j=k$ :

$$
b_{k}=\frac{x_{k}}{x_{k+1}} \frac{P_{k}\left(\lambda^{(n)}\right)}{P_{k-1}\left(\lambda^{(n)}\right)} .
$$


Then, from (3.6),

$$
\begin{aligned}
b_{k+1} & =\left(\lambda^{(n)}-a_{k+1}\right) \frac{x_{k+1}}{x_{k+2}}-b_{k} \frac{x_{k}}{x_{k+2}} \\
& =\left(\lambda^{(n)}-a_{k+1}\right) \frac{x_{k+1}}{x_{k+2}}-b_{k} \frac{x_{k}}{x_{k+2}}\left[\frac{x_{k}}{x_{k+1}} \frac{P_{k}\left(\lambda^{(n)}\right)}{P_{k-1}\left(\lambda^{(n)}\right)} \frac{x_{k+1}}{x_{k}} \frac{P_{k-1}\left(\lambda^{(n)}\right)}{P_{k}\left(\lambda^{(n)}\right)}\right] \\
& =\left(\lambda^{(n)}-a_{k+1}\right) \frac{x_{k+1}}{x_{k+2}}-b_{k} \frac{x_{k}}{x_{k+2}}\left[b_{k} \frac{x_{k+1}}{x_{k}} \frac{P_{k-1}\left(\lambda^{(n)}\right)}{P_{k}\left(\lambda^{(n)}\right)}\right] \\
& =\frac{x_{k+1}}{x_{k+2}}\left[\left(\lambda^{(n)}-a_{k+1}\right)-b_{k}^{2} \frac{P_{k-1}\left(\lambda^{(n)}\right)}{P_{k}\left(\lambda^{(n)}\right)}\right] \\
& =\frac{x_{k+1}}{x_{k+2}}\left[\frac{\left(\lambda^{(n)}-a_{k+1}\right) P_{k}\left(\lambda^{(n)}\right)-b_{k}^{2} P_{k-1}\left(\lambda^{(n)}\right)}{P_{k}\left(\lambda^{(n)}\right)}\right] \\
& =\frac{x_{k+1}}{x_{k+2}} \frac{P_{k+1}\left(\lambda^{(n)}\right)}{P_{k}\left(\lambda^{(n)}\right)} .
\end{aligned}
$$

Thus, (3.7) holds and

$$
x_{n-1}=b_{n-1} \frac{P_{n-2}\left(\lambda^{(n)}\right)}{P_{n-1}\left(\lambda^{(n)}\right)} .
$$

Now,

$$
\begin{aligned}
b_{n-1} x_{n-1}+a_{n} x_{n} & =b_{n-1} x_{n-1}+\left[\lambda^{(n)}-b_{n-1}^{2} \frac{P_{n-2}\left(\lambda^{(n)}\right)}{P_{n-1}\left(\lambda^{(n)}\right)}\right] x_{n} \\
& =b_{n-1} x_{n-1}+\lambda^{(n)} x_{n}-b_{n-1}\left[b_{n-1} \frac{P_{n-2}\left(\lambda^{(n)}\right)}{P_{n-1}\left(\lambda^{(n)}\right)}\right] x_{n} \\
& =b_{n-1} x_{n-1}+\lambda^{(n)} x_{n}-b_{n-1} x_{n-1} \\
& =\lambda^{(n)} x_{n},
\end{aligned}
$$

and the last equation in (3.5) is true.

Hence, there exists $A$ of the form (1.3), with positive entries $b_{i}, \lambda^{(j)}$ being an eigenvalue of the leading principal matrix $A_{j}, j=1,2, \ldots, n$ and $\left(\lambda^{(n)}, \mathbf{x}\right)$ an eigenpair of $A$. प 
REMARK 3.3. Theorem 3.2 also holds if we take $\lambda^{(j)}$ as the minimal eigenvalue of $A_{j}$. In this case we must have $\lambda^{(n)}<\cdots<\lambda^{(2)}<\lambda^{(1)}$ and condition (3.1) become $x_{i} x_{i+1}<0, i=1, \ldots, n$. The proof is completely similar.

Now we give sufficient conditions in order that the solution matrix in Problem 1.2 (with $\lambda^{(j)}$ as the minimal eigenvalue of $A_{j}$ ) be nonnegative.

Corollary 3.4. Let the $n$ real numbers $\lambda^{(1)}<\lambda^{(2)}<\cdots<\lambda^{(n)}$, and a real vector $\mathbf{x}=\left(x_{1}, \ldots, x_{n}\right)^{T}$ be given. If

$$
\begin{gathered}
x_{i} x_{i+1}<0, \quad i=1,2, \ldots, n-1, \\
\lambda^{(n)}>a_{j}+b_{j-1} \frac{x_{j-1}}{x_{j}}, \quad j=2,3, \ldots, n-1,
\end{gathered}
$$

and

$$
\lambda^{(1)} \geq 0 \quad \text { and } \quad \lambda^{(j)} \geq b_{j-1}^{2} \frac{P_{j-2}\left(\lambda^{(j)}\right)}{P_{j-1}\left(\lambda^{(j)}\right)}, \quad j=1,2, \ldots, n
$$

then there exists an $n \times n$ nonnegative matrix $A$ of the form (1.3), such that $\lambda^{(j)}$ is the maximal eigenvalue of the $j \times j$ leading principal submatrix $A_{j}$ of $A, j=1,2, \ldots, n$ and $\left(\lambda^{(n)}, \mathbf{x}\right)$ is an eigenpair of $A$.

Proof. From Theorem 3.2, conditions (3.8) and (3.9) guarantee the existence of $A$ of the form (1.3) with positive entries $b_{i}, \lambda^{(j)}$ being the maximal eigenvalue of $A_{j}$, and $\left(\lambda^{(n)}, \mathbf{x}\right)$ an eigenpair of $A$. It only remains to show the nonnegativity of the diagonal entries $a_{j}, j=1,2, \ldots, n$. From (3.10), it follows that $a_{1}=\lambda^{(1)} \geq 0$ and

$$
a_{j}=\lambda^{(j)}-b_{j-1}^{2} \frac{P_{j-2}\left(\lambda^{(j)}\right)}{P_{j-1}\left(\lambda^{(j)}\right)} \geq 0, \quad j=2,3, \ldots, n .
$$

\section{Examples.}

EXAMPLE 4.1. The real numbers

$$
\begin{array}{cccccc}
\lambda^{(1)} & \lambda^{(2)} & \lambda^{(3)} & \lambda^{(4)} & \lambda^{(5)} & \lambda^{(6)} \\
3.7542 & 4.4013 & 9.7618 & 10.2563 & 11.5778 & 11.5789
\end{array}
$$

and the real vector

$$
\mathbf{x}=\left[\begin{array}{llllll}
-0.6942 & -0.0742 & -0.5200 & -0.2501 & -0.4237 & -0.0123
\end{array}\right]^{T}
$$


satisfy conditions of Corollary 2.8. Then we may construct the nonnegative symmetric arrow matrix

$$
A=\left[\begin{array}{llllll}
3.7542 & 0.9006 & 6.1056 & 1.9487 & 4.0160 & 0.1232 \\
0.9006 & 3.1479 & & & & \\
6.1056 & & 3.4273 & & & \\
1.9487 & & & 6.1696 & & \\
4.0160 & & & & 4.9990 & \\
0.1232 & & & & & 4.6291
\end{array}\right]
$$

where the spectra of the matrices $A_{j}$ are $\sigma\left(A_{1}\right)=\{\mathbf{3 . 7 5 4 2}\}$,

$\sigma\left(A_{2}\right)=\left\{\begin{array}{ll}2.5008 & \mathbf{4 . 4 0 1 3}\end{array}\right\}$,

$\sigma\left(A_{3}\right)=\left\{\begin{array}{lll}-2.5863 & 3.1539 & \mathbf{9} .7618\end{array}\right\}$,

$\sigma\left(A_{4}\right)=\left\{\begin{array}{llll}-2.7960 & 3.1538 & 5.8848 & \mathbf{1 0 . 2 5 6 3}\end{array}\right\}$,

$\sigma\left(A_{5}\right)=\left\{\begin{array}{lllll}-3.7669 & 3.1535 & 4.5001 & 6.0335 & \mathbf{1 1 . 5 7 7 8}\end{array}\right\}$,

$\sigma\left(A_{6}\right)=\left\{\begin{array}{llllll}-3.7678 & 3.1535 & 4.4989 & 4.6301 & 6.0335 & \mathbf{1 1 . 5 7 8 9}\end{array}\right\}$,

and $A \mathbf{x}=(11.5789) \mathbf{x}$.

EXAMPLE 4.2. Now we consider the following spectral information:

$$
\begin{array}{cccccc}
\lambda^{(1)} & \lambda^{(2)} & \lambda^{(3)} & \lambda^{(4)} & \lambda^{(5)} & \lambda^{(6)} \\
3.7542 & 2.5008 & -2.5863 & -2.7960 & -3.7669 & -3.7678
\end{array}
$$

and

$$
\mathbf{x}=\left[\begin{array}{llllll}
-0.7097 & 0.0924 & 0.6022 & 0.1392 & 0.3251 & 0.0104
\end{array}\right]^{T} .
$$

Then, we obtain

$$
A=\left[\begin{array}{llllll}
3.7542 & 0.9006 & 6.1056 & 1.9487 & 4.0160 & 0.1232 \\
0.9006 & 3.1479 & & & & \\
6.1056 & & 3.4273 & & & \\
1.9487 & & & 6.1696 & & \\
4.0160 & & & & 4.9990 & \\
0.1232 & & & & & 4.6291
\end{array}\right]
$$

where

$$
\begin{aligned}
& \sigma\left(A_{1}\right)=\left\{\begin{array}{ll}
3.7542
\end{array}\right\}, \\
& \sigma\left(A_{2}\right)=\left\{\begin{array}{ll}
2.5008 & 4.4013
\end{array}\right\},
\end{aligned}
$$


$\sigma\left(A_{3}\right)=\left\{\begin{array}{lll}\mathbf{- 2 . 5 8 6 3} & 3.1539 & 9.7618\end{array}\right\}$,

$\sigma\left(A_{4}\right)=\left\{\begin{array}{llll}\mathbf{- 2 . 7 9 6 0} & 3.1538 & 5.8848 & 10.2563\end{array}\right\}$,

$\sigma\left(A_{5}\right)=\left\{\begin{array}{lllll}-\mathbf{3 . 7 6 6 9} & 3.1535 & 4.5001 & 6.0335 & 11.5778\end{array}\right\}$,

$\sigma\left(A_{6}\right)=\left\{\begin{array}{llllll}-\mathbf{3 . 7 6 7 8} & 3.1535 & 4.4989 & 4.6301 & 6.0335 & 11.5789\end{array}\right\}$,

and $A \mathbf{x}=(-3.7678) \mathbf{x}$.

EXAMPLE 4.3. Given

$$
\begin{array}{ccccccc}
\lambda^{(1)} & \lambda^{(2)} & \lambda^{(3)} & \lambda^{(4)} & \lambda^{(5)} & \lambda^{(6)} & \lambda^{(7)} \\
0.6829 & 3.4716 & 4.4172 & 4.4194 & 4.4195 & 4.4198 & 4.4238
\end{array}
$$

and the vector

$$
\mathbf{x}=\left[\begin{array}{lllllll}
0.2275 & 0.5257 & 0.4566 & 0.0532 & 0.1407 & 0.1829 & 0.6383
\end{array}\right]^{T},
$$

we construct the nonnegative symmetric tridiagonal matrix

$$
A=\left[\begin{array}{lllllll}
0.6829 & 1.6186 & & & & & \\
1.6186 & 2.5321 & 1.3716 & & & & \\
& 1.3716 & 2.8277 & 0.1452 & & & \\
& & 0.1452 & 0.6665 & 0.9498 & & \\
& & & 0.9498 & 0.3571 & 2.8518 & \\
& & & & 2.8518 & 1.0901 & 0.3266 \\
& & & & & 0.3266 & 4.3302
\end{array}\right],
$$

with the required spectral properties, where

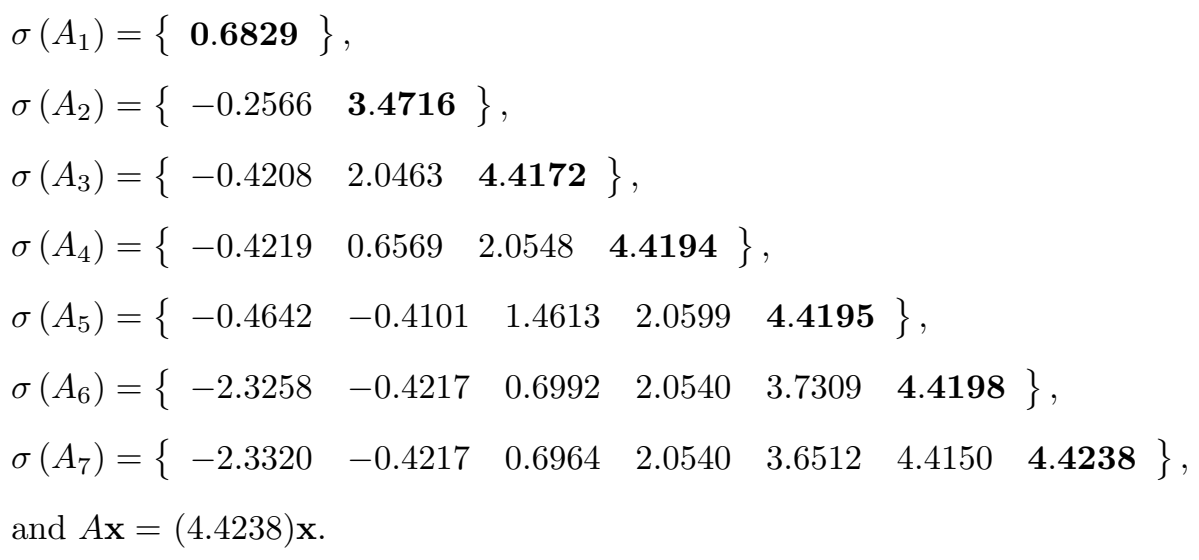




\section{REFERENCES}

[1] G.W. Bing. Introduction to the Nonlinear Control Systems. Science Press, 1988.

[2] D. Boley and G.H. Golub. A survey of matrix inverse eigenvalue problem. Inverse Problems, 3:595-622, 1987.

[3] M.T. Chu and G.H. Golub. Inverse Eigenvalue Problems: Theory, Algorithms and Applications. Oxford University Press, New York, 2005.

[4] J.C. Egaña, N.M. Kuhl, and L.C. Santos. An inverse eigenvalue method for frequency isolation in spring-mass systems. Numer. Linear Algebra Appl., 9:65-79, 2002.

[5] G.M. Gladwell. Inverse Problems in Vibration, 2nd edition. Kluwer Academic Publishers, Dordrecht, 2004.

[6] X.Y. Hu, L. Zhang, and Z.Y. Peng. On the construction of a Jacobi matrix from its defective eigenpairs and a principal submatrix. Math. Numer. Sinca, 3:345-354, 2000.

[7] X. Huang, X. Hu, and L. Zhang. Physical parameters reconstruction of a fixed-fixed mass-spring system from its characteristic data. J. Comput. Appl. Math., 206:645-655, 2007.

[8] J. Peng, X.Y. Hu, and L. Zhang. Two inverse eigenvalue problems for a special kind of matrices. Linear Algebra Appl., 416:336-347, 2006.

[9] H. Pickmann, J. Egaña, and R.L. Soto. Extremal inverse eigenvalue problem for bordered diagonal matrices. Linear Algebra Appl., 427:256-271, 2007.

[10] H. Pickmann, R.L. Soto, J. Egaña, and M. Salas. An inverse eigenvalue problem for symmetrical tridiagonal matrices. Comput. Math. Appl., 54(5):699-708, 2007. 